\title{
Transitorios hidráulicos generados por arranque de equipos de bombeo en conducciones a presión. Aplicación a un caso real
}

\author{
L.G. Carmona-Paredes y R.B. Carmona-Paredes \\ Instituto de Ingeniería, UNAM \\ Email:lcp@pumas.iingen.unam.mx,yrcp@pumas.iingen.unam.mx \\ (recibido: marzo de 1999; aceptado: agosto de 2000)
}

\begin{abstract}
Resumen
Al diseñar conducciones a presión, es común que se preste especial atención a los transitorios hidráulicos generados por paros de emergencia de los equipos de bombeo. En el acueducto río YaquiGuaymas, Sonora, México, los transitorios generados al poner en marcha los equipos de bombeo son tan importantes como los producidos por disparo de bombas. En el Instituto de Ingeniería de la UNAM, se desarrolló e implantó en su programa de simulación de transitorios hidráulicos en tuberías a presión una subrutina para simular el arranque de equipos de bombeo. El modelo matemático está formado por el conjunto de ecuaciones dinámica y de continuidad, así como la condición de frontera que describe el arranque de las bombas. Se reescriben las ecuaciones dinámica y de continuidad, con el método de las características y se plantea un sistema de ecuaciones no lineales, el cual se resuelve con el método de Newton-Raphson. La simulación del arranque de las bombas del acueducto en estudio, arroja valores de las cargas piezométricas máximas y mínimas mayores que la resistencia de la tubería y menores que la topografía del terreno, respectivamente en las zonas de falla de la tubería, lo que concuerda con los problemas observados en campo. Los resultados se muestran en las gráficas que se incluyen.
\end{abstract}

Descriptores: transitorios hidráulicos, plantas de bombeo, conducciones a presión, ruptura de tuberías, método de las características.

\begin{abstract}
Water closed conduits are commonly designed with special attention paid to pressure surges due to pumping failures. In some particular cases, such at the Rio Yaqui-Guaymas water pipeline system, pressure surges due to pumping start ups are as high and dangerous as those produced by pumping failures. New subroutines were thus developed and included in the Closed Conduit Flow Transient Model developed by the Instituto de Ingeniería of the Universidad Nacional Autónoma de México. This paper presents the mathematical model and its numerical solution using the Method of Characteristics and the Newton-Raphson Method. With the new modeling system it is possible to evaluate the pressure fluctuation time series due to start up at any pumping system. Using this new system a reliable explanation of bursts at the Rio Yaqui-Guaymas pipeline system could be set forth.
\end{abstract}

Keywords: pressure surge, pumping systems, pipeline systems, bursts, method of characteristics.

\section{Introducción}

Se presenta un modelo numérico de simulación del transitorio hidráulico en tuberías a presión, generado por el arranque de equipos de bombeo y su aplicación al acueducto río Yaqui-Guaymas, Sonora, México.
El arranque de las bombas en una estación de bombeo produce en la conducción un transitorio hidráulico que se refleja en la variación de la carga piezométrica en la tubería.

Los cálculos de la variación de la carga piezométrica se efectúan agregando a las ecuaciones dinámica y de continuidad la descripción de las bombas como condición de frontera. 
DOI: http://dx.doi.org/10.22201/fi.25940732e.2000.01n5.022

Usando el método de las características para rescribir las ecuaciones de continuidad y dinámica, así como la condición de frontera que imponen las bombas, se construye el modelo matemático que representa el transitorio hidráulico en tubería a presión, generado por el arranque de bombas.

\section{Modelo matemático de las bombas}

El modelo matemático del arranque de equipos de bombeo que se implantó incluye dos situaciones: cuando no hay ninguna bomba en operación y se ponen en marcha algunos equipos, o bien, cuando se incrementa el número de bombas estando algunas funcionando previamente.

Se supone que la variación de la velocidad de giro de las bomba es lineal en el tiempo.

\section{Arranque de la primera bomba}

El sistema de ecuaciones que modela el transitorio producido por el arranque del primer equipo de bombeo está formado por las siguientes ecuaciones:

Ecuación dinámica del flujo a través de las válvulas de descarga:

$$
H_{P_{i+1, i}}=H_{p_{i, n+1}}+\bar{h}_{p} H_{R}-\bar{c} v Q_{R}^{2} \bar{v} p|\bar{v} p|
$$

Ecuación de continuidad:

$$
Q_{P_{i+1,1}}=Q_{P_{i n+1}}=Q_{R} \bar{v}_{P}
$$

Ecuación de la característica negativa en la succión de las bombas:

$$
\begin{gathered}
Q_{P_{i+1,1}}=c_{n}+c a_{i+1} H_{P_{i+1,1}} \text { donde: } \\
C_{n}=Q_{B}-\frac{g A}{a} H_{B}-\frac{f \Delta t}{2 D A} Q_{B}\left|Q_{B}\right| \text { y } c a_{i+1}=\frac{g A_{i+1}}{a_{i+1}}
\end{gathered}
$$

Ecuación de la característica positiva en la descarga de las bombas:

$$
\begin{gathered}
Q_{P_{i, n+1}}=c_{p}-c a_{i} H_{P_{i n+1}} \text { donde } \\
C_{p}=Q_{A}+\frac{g A}{a} H_{A}-\frac{f \Delta t}{2 D A} Q_{A}\left|Q_{A}\right| \text { y } c a_{i}=\frac{g A_{i}}{a_{i}}
\end{gathered}
$$

Y ecuaciones que modelan las bombas. Punto de operación de las bombas:

$$
\frac{\bar{h}_{\mathrm{p}}}{\bar{\alpha}_{P}^{2}+\bar{v}_{P}^{2}}=\bar{a}_{1}+\bar{a}_{2} \text { angtan } \frac{\bar{\alpha}_{P}}{\bar{v}_{P}} ;
$$

donde $\bar{a}_{1}$ y $\bar{a}_{2}$ son parámetros para obtener el punto de operación de las bombas que se obtienen como se indica en Chaudhry (1979), capítulo 4.

Variación de la velocidad de giro de las bombas:

$$
\bar{\alpha}_{P}=\frac{t-t_{a}}{t_{b}}
$$

Las incógnitas del sistema de ecuaciones 1 a 6 son:

$$
H_{P_{i+1,1}}, H_{P_{i, n+1}}, Q_{P_{i+1,1}}, \bar{h}_{P}, \bar{v}_{P} \text { y } \bar{\alpha}_{P} .
$$

Los subíndices $(i, n+1),(i+1,1)$ representan los puntos de cálculo del método de las características localizados aguas arriba y aguas abajo de las bombas, respectivamente.

El sistema formado por las ecuaciones 1 a 6 , se reduce a la ecuación:

$$
\begin{gathered}
H_{R}\left(\bar{\alpha}_{P}^{2}+\bar{v}_{P}^{2}\right)\left(\bar{a}_{1}+\bar{a}_{2} \operatorname{angtan} \frac{\bar{\alpha}_{P}}{\bar{v}_{P}}\right)-\bar{c}_{v} Q_{R}^{2} \bar{v}_{P}\left|\bar{v}_{P}\right|-Q_{R} \\
\left(\frac{1}{c a_{i}}+\frac{1}{c a_{i+1}}\right) \bar{v}_{P}+\frac{c_{n}}{c a_{i+1}}+\frac{c_{P}}{c a_{i}}=0
\end{gathered}
$$

cuya incógnita es el gasto adimensionado $\bar{v}_{p}$. Resolviendo la ecuación 7 con el método iterativo de Newton-Raphson, se obtiene que

$$
\bar{v}_{P}=\bar{v}-\frac{F(\bar{v})}{F^{\prime}(\bar{v})}
$$

donde

$$
\begin{gathered}
F(\bar{v})=H_{R}\left(\bar{\alpha}_{P}^{2}+\bar{v}^{2}\right)\left(\bar{a}_{1}+\bar{a}_{2} \text { angtan } \frac{\bar{\alpha}_{P}}{\bar{v}}\right)-\bar{c}_{v} Q_{R}^{2} \bar{v}|\bar{v}|-Q_{R} \\
\left(\frac{1}{c a_{i}}+\frac{1}{c a_{i+1}}\right) \bar{v}+\frac{c_{n}}{c a_{i+1}}+\frac{c_{P}}{c a_{i}} \\
F^{\prime}(\bar{v})=2 H_{R} \bar{v}\left(\bar{a}_{1}+\bar{a}_{2} \text { angtan } \frac{\bar{\alpha}_{P}}{\bar{v}}\right)- \\
H_{R} \bar{a}_{2} \bar{\alpha}_{P}-\overline{2}_{v} Q_{R}^{2}|\bar{v}|-Q_{R}\left(\frac{1}{c a_{i}}+\frac{1}{c a_{i+1}}\right)
\end{gathered}
$$

es la derivada de $F(\bar{v})$ con respecto a $\bar{v}, \bar{\alpha}_{P}$ es una función conocida dada por 6 y $\bar{v}$ es el valor del gasto adimensionado en el instante de cálculo anterior al actual, para $t=0$ es el gasto en estado estacionario.

El algoritmo para resolver la ecuación no lineal 7 es:

1) Tomar el valor inicial para $\bar{v}$.

2) Calcular $\bar{\alpha}_{p}$ con la ecuación 6 . 
3) Calcular los parámetros, $\bar{a}_{1}$ y $\bar{a}_{2}$, según se indica en Chaudhry (1979).

4) Evaluar las funciones $F$ y $F^{\prime}$ con las ecuaciones 9 y 10.

5) Calcular el nuevo valor de $\bar{v}_{\mathrm{p}}$ con la ecuación 8 .

6) $\mathrm{Si}\left|\bar{v}-\bar{v}_{\mathrm{P}}\right|<$ tolerancia entonces, termina el algoritmo para el instante de cálculo t. En caso contrario, hacer $\bar{v}=\bar{v}_{p}$ y regresar al punto 3 .

7) Con el valor obtenido para $\bar{v}_{p}$, se evalúan las demás incógnitas a través de las ecuaciones 1 a 6 .

8) Si $t>$ tiempo de observación, termina el algoritmo, si no se regresa al inciso 1 con el valor $\bar{v}=\bar{v}_{P}$ hasta que $t>$ tiempo de observación.

\section{Arranque de una bomba cuando algunas están operando}

Para completar, respecto al caso anterior, el modelo matemático del funcionamiento transitorio del arranque de una bomba cuando hay algunas operando, se deberán agregar al sistema de ecuaciones formado por las ecuaciones 1 a 6 , las siguientes ecuaciones correspondientes a la descripción de las bombas que ya están en operación. Notación, las variables sin barra se refieren a las bombas que ya están en operación y las variables con barra a las bombas que van arrancar.

$$
H_{P_{i+1, i}}=H_{P_{i, n+1}}+h_{p} H_{R}-c_{v} Q_{R}^{2} v_{P}\left|v_{P}\right|
$$

y

$$
\frac{h_{\mathrm{P}}}{1+v_{\mathrm{P}}^{2}}=a_{1}+a_{2} \operatorname{angtan} \frac{1}{v_{P}}
$$

La ecuación de continuidad 2 debe cambiarse por la siguiente ecuación

$$
Q_{P_{i+1,1}}=Q_{P_{i, n+1}}=N_{B} Q_{R} v_{P}+Q_{R} \bar{v}_{P}
$$

Entonces, se obtiene un sistema no lineal de ocho ecuaciones formado por las ecuaciones 1,3 a $6,11,12$ y 13 , cuyas incógnitas son $H_{P_{i+1,1}}, H_{P_{i, n+1}}, Q_{P_{i+1,1}}, \bar{h}_{\mathrm{p}}, \bar{v}_{\mathrm{p}}, \bar{\alpha}_{\mathrm{p}}, h_{p}$ y $v_{\mathrm{p}}$.

El sistema de ecuaciones formado por (1), (3) a (6) y (11) a (13) se puede reducir al siguiente sistema de dos ecuaciones no lineales con variables $v_{\mathrm{p}}$ y $\bar{v}_{\mathrm{p}}$, ya que

$$
\begin{gathered}
\bar{\alpha}_{P}=\frac{t-t_{u}}{t_{b}} \\
H_{R}\left(\bar{\alpha}_{P}^{2}+\bar{v}_{P}^{2}\right)\left(\bar{a}_{1}+\bar{a}_{2} \operatorname{angtan} \frac{\bar{\alpha}_{P}}{\bar{v}_{P}}\right)-\bar{c}_{v} Q_{R}^{2} \bar{v}_{P}\left|\bar{v}_{P}\right|-Q_{R} \\
\left(N_{B} v_{P}+\bar{v}_{P}\right)\left(\frac{1}{c a_{i}}+\frac{1}{c a_{i+1}}\right)+\frac{c_{p}}{c a_{i}}+\frac{c_{n}}{c a_{i+1}}=0
\end{gathered}
$$

$$
\begin{gathered}
H_{R}\left(1+v_{P}^{2}\right)\left(a_{1}+a_{2} \operatorname{angtan} \frac{1}{v_{P}}\right)-c_{v} Q_{R}^{2} v_{P}\left|v_{P}\right|-Q_{R} \\
\left(N_{B} v_{P}+\bar{v}_{P}\right)\left(\frac{1}{c a_{i}}+\frac{1}{c a_{i+1}}\right)+\frac{c_{p}}{c a_{i}}+\frac{c_{n}}{c a_{i+1}}=0
\end{gathered}
$$

El sistema de ecuaciones no lineales acopladas formado por (14) y (15) se puede resolver con el método iterativo de Newton-Raphson. Definiendo

$$
\begin{gathered}
F_{1}=H_{R}\left(\bar{\alpha}_{P}^{2}+\bar{v}_{P}^{2}\right)\left(\bar{a}_{1}+\bar{a}_{2} \text { angtan } \frac{\bar{\alpha}_{P}}{\bar{v}_{P}}\right)-\bar{c}_{v} Q_{R}^{2} \bar{v}_{P}\left|\bar{v}_{P}\right|-Q_{R} \\
\left(N_{B} v_{P}+\bar{v}_{P}\right)\left(\frac{1}{c a_{i}}+\frac{1}{c a_{i+1}}\right)+\frac{c_{p}}{c a_{i}}+\frac{c_{n}}{c a_{i+1}} \\
F_{2}=H_{R}\left(1+v_{P}^{2}\right)\left(a_{1}+a_{2} \operatorname{angtan} \frac{1}{v_{P}}\right)-c_{v} Q_{R}^{2} v_{P}\left|v_{P}\right|-Q_{R} \\
\left(N_{B} v_{P}+\bar{v}_{P}\right)\left(\frac{1}{c a_{i}}+\frac{1}{c a_{i+1}}\right)+\frac{c_{p}}{c a_{i}}+\frac{c_{n}}{c a_{i+1}}
\end{gathered}
$$

Las soluciones para $v_{\mathrm{P}}$ y $\bar{v}_{\mathrm{P}}$, están dadas por

$$
v_{P}=v+\frac{-F_{1} F_{2 \bar{v}}+F_{2} F_{1 \bar{v}}}{F_{1 v} F_{2 \bar{v}}-F_{1 \bar{v}}-F_{2 v}} \text { y } \quad \bar{v}_{P}=\bar{v}+\frac{-F_{1 v} F_{2}+F_{2 v} F_{1}}{F_{1 v} F_{2 \bar{v}}-F_{1 \bar{v}} F_{2 v}}
$$

donde, los subíndices $v$ y $\bar{v}$ denotan derivadas parciales de las funciones $F_{1}$ y $F_{2}$ respecto a $v$ y $\bar{v}$.

El algoritmo para resolver el sistema anterior de ecuaciones es el siguiente:

1) Hacer $v_{P}=v$ y $\bar{v}_{P}=\bar{v}$. Para $t=0, v$ y $\bar{v}$ son los valores del gasto adimensionado en el estado estacionario para las bombas en operación y las que van a arrancar, respectivamente.

2) Calcular $\bar{\alpha}_{p}$ con la ecuación 6 .

3) Calcular los parámetros $a_{1}, a_{2}, \bar{a}_{1}$ y $\bar{a}_{2}$ como se indica en Chaudhry (1979), capítulo 4.

4) Evaluar las funciones $F_{1}$ y $F_{2}$ con las ecuaciones 16 y 17.

5) Evaluar las derivadas parciales de $F_{1}$ y $F_{2}$.

6) Calcular los nuevos valores de $v_{P}$ y $\bar{v}_{P}$ con las ecuaciones 18 .

7) Si $\left|\bar{v}-\bar{v}_{p}\right|<$ tolerancia y $\left|\nu-v_{p}\right|<$ tolerancia entonces, termina el algoritmo para el instante de cálculo t. En caso contrario, regresar al punto 1). 
DOI: http://dx.doi.org/10.22201/fi.25940732e.2000.01n5.022

Con los valores obtenidos para $\bar{v}_{P}$ y $v_{\mathrm{P}}$ se evalúan las demás incógnitas a través de las ecuaciones 1,3 a 5 y 11 a 13 .

8) Si $t<$ tiempo de observación, se regresa al inciso 1 , si no termina el algoritmo.

\section{Resultados numéricos}

El acueducto río Yaqui-Guaymas se diseñó para conducir, en una tubería de 36" de diámetro y longitud aproximada de $100 \mathrm{~km}, 520 \mathrm{lt} / \mathrm{s}$ por gravedad u $850 \mathrm{lt} / \mathrm{s}$ por inyección forzada con dos plantas de bombeo. El aprovechamiento es una batería de pozos que envía el agua a un tanque cambio de régimen, a partir del cual inicia el tramo en estudio.

El perfil topográfico del acueducto se muestra en la figura 1. El cadenamiento corre en sentido contrario al flujo. El tanque de cambio de régimen se localiza en el cadenamiento $100+000$ con elevación de $60 \mathrm{~m}$. La planta de bombeo 1 (PB1) se ubica en el cadenamiento $72+912$ y elevación 27 m y la PB2 en el $23+251$ con elevación 13 m. El origen del cadenamiento está en el tanque de entrega en la localidad de Empalme.

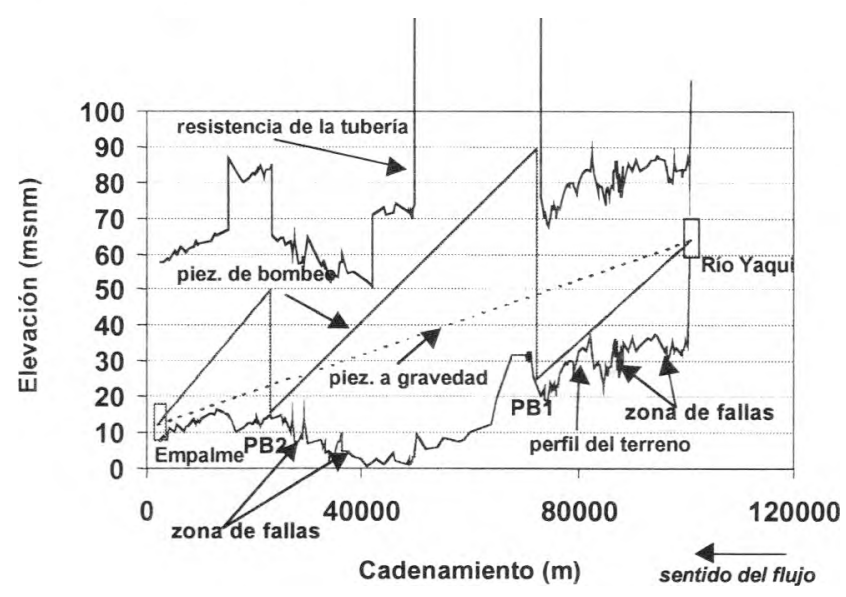

Figura 1. Cargas piezométricas de operación a gravedad $(Q=500 \mathrm{lt} / \mathrm{s})$ y por bombeo $(Q=850 \mathrm{lt} / \mathrm{s})$

En la misma figura se muestran las cargas piezométricas de operación para $500 \mathrm{lt} / \mathrm{s}$ por gravedad y para $850 \mathrm{lt} / \mathrm{s}$ por bombeo.

El arreglo funcional de las plantas de bombeo es como se muestra en la figura 2.

Durante la operación por gravedad del acueducto, el agua fluye a través de las baterías de válvulas check desde el extremo inicial del acueducto hasta el punto de entrega. $\mathrm{Al}$ arrancar el primer equipo de bombeo en PB1, aumenta rápidamente la presión en la tubería aguas abajo de la batería de válvulas check, lo que produce su cierre instantáneo y genera un golpe de ariete (sobrepresión) que viaja en contra del sentido de flujo de agua. El incremento súbito de presión por el arranque de la bomba genera también un transitorio de sobrepresión que viaja en sentido del flujo, desde el sitio de la planta de bombeo 1, hasta PB2.

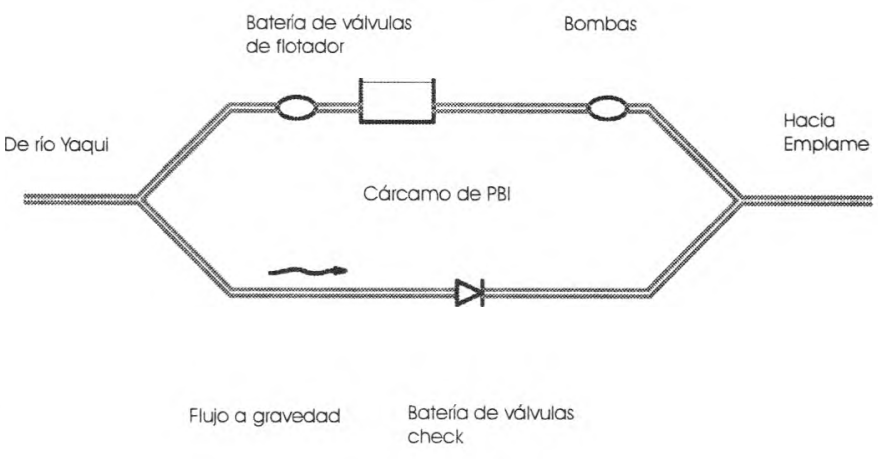

Figura 2. Cargas piezométricas de operación a gravedad $(Q=500 \mathrm{lt} / \mathrm{s})$ y por bombeo $(Q=850 \mathrm{lt} / \mathrm{s})$

Los reflejos de las perturbaciones de sobrepresión, en el tanque de cambio de régimen para la onda que viaja en sentido contrario al flujo, y en el cárcamo de PB2 para la que viaja en sentido del flujo, generan perturbaciones de baja presión capaces de establecer presiones negativas y succión de aire en algunos tramos de la conducción.

La figura 3, muestra las cargas piezométricas máximas y mínimas generadas por el arranque del primer equipo de bombeo en la planta 1, la topografía del terreno y la resistencia de la tubería instalada. Esta simulación se realizó suponiendo que las válvulas de flotador colocadas a la llegada del cárcamo de PB1, ver figura 2, estaban cerradas durante el arranque.

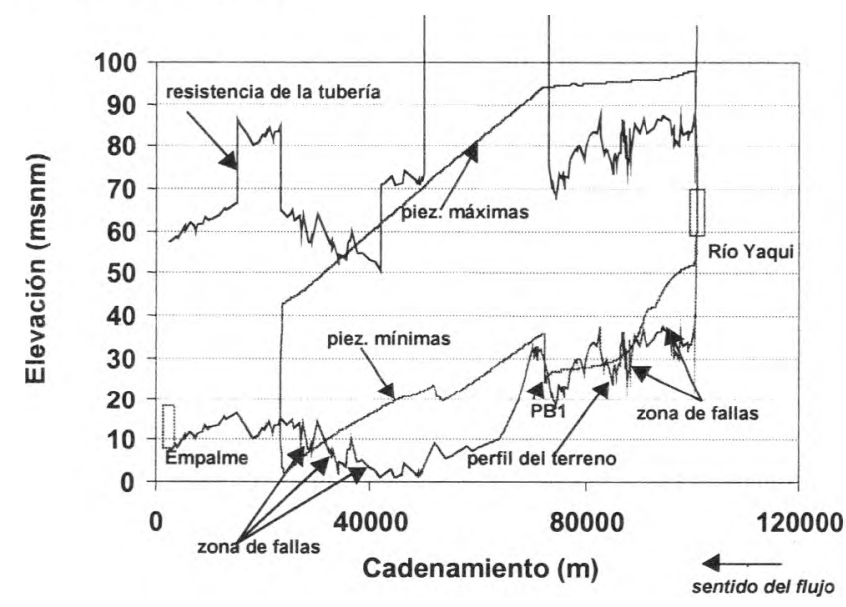

Figura 3. Cargas piezométricas máximas y minimas durante el arranque de una bomba booster con el cárcamo de bombeo cerrado. No hay dispositivos de control de transitorios 
Se observa cómo el transitorio genera presiones mayores que la capacidad de resistencia de la tubería desde la zona de pozos de río Yaqui hasta la planta de bombeo y alrededor del cadenamiento $40+000$. En esa misma gráfica se indican las zonas en donde se reportaron fallas en la tubería durante los arranques.

En la figura 4 se muestran las cargas piezométricas generadas durante el transitorio de arranque del primer equipo de la planta 1 suponiendo que las válvulas de altitud a la llegada del cárcamo, están abiertas (Figura 2). Se observa que las presiones máximas generadas no son tan altas como las del caso anterior, sin embargo, se producen presiones mayores que la resistencia de la tubería, alrededor del cadenamiento $40+000$ y depresiones en el tramo que va de la zona de pozos a la planta de bombeo 1 .

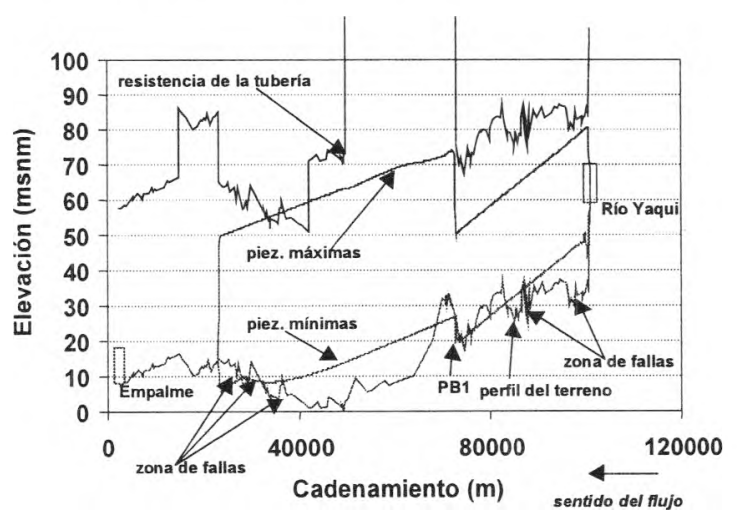

Figura 4. Cargas piezométricas máximas y mínimas durante el arranque de una bomba booster con el cárcamo de bombeo abierto. Sin dispositivos de control de transitorios

\section{Conclusiones}

La representación de transitorios hidráulicos por arranque de bombas con el modelo que se presenta en este artículo, ha permitido identificar el origen de las fallas reportadas durante las pruebas de puesta en servicio de las estaciones de bombeo en el acueducto río Yaqui-Guaymas.
En combinación con las otras opciones del programa de simulación de transitorios del Instituto de Ingeniería, se ha propuesto un arreglo de dispositivos de control de transitorios hidráulicos para evitar que las oscilaciones de presión por arranque de bombas superen la resistencia de la tubería y propicien succión de aire.

Debido a la creciente demanda de agua en muchas ciudades de nuestro país, el uso de rebombeos será cada vez más frecuente, por lo que el problema que aquí se discute será base importante para la ampliación de la capacidad de conducción de sistemas existentes.

\section{Nomenclatura}

$\bar{\alpha}_{p} \quad$ velocidad de giro de la bomba que se arranca

$\mathrm{t}_{\mathrm{a}} \quad$ instante de arranque de las bombas

$t_{b}$ tiempo que tarda el motor en alcanzar su velocidad nominal

$t$ tiempo

$\mathrm{H}_{\mathrm{P}}$ carga en el instante $t$

$Q_{\mathrm{P}}$ gasto en el instante $t$

$\mathrm{H}_{\mathrm{R}}$ carga nominal de las bombas

$Q_{R}$ gasto nominal de las bombas

$h_{\mathrm{P}}=\mathrm{H}_{\mathrm{P}} / \mathrm{H}_{\mathrm{R}}$ carga adimensional en el instante $t$

$v_{\mathrm{P}}=\mathrm{Q}_{\mathrm{P}} / \mathrm{Q}_{\mathrm{R}}$ gasto adimensional en el instante $t$

$v=Q / Q_{R} \quad$ gasto adimensional en el instante $t-\Delta t$

$\mathrm{C}_{v} \quad$ coeficiente de pérdida de las válvulas ubicadas en la descarga de las bombas

g aceleración de la gravedad

A área de la conducción

a velocidad de la onda de presión

f factor de fricción

D diámetro de la conducción

$\Delta t \quad$ intervalo integración en el tiempo

$\mathrm{N}_{\mathrm{B}}$ número de bombas

\section{Referencia}

Chaudhry M.H. (1979). Applied Hydraulic Transients. Van Nostrand Reinhold. New York, USA.

\section{Semblanza de los autores}

Libia Carmona-Paredes. Estudió la carrera de física en la Facultad de Ciencias de la UNAM. Trabaja en el Instituto de Ingeniería (II) de la UNAM en la Coordinación de Mecánica, Térmica y Fluidos. Desde su ingreso al II ha trabajado en el diseño de modelos numéricos y su implantación en sistemas de cómputo. Entre los resultados de su trabajo destaca el sistema de simulación de transitorios hidráulicos en tuberías a presión, herramienta ampliamente utilizada en el diseño y estudio de los sistemas de conducción a presión de agua potable en el país.

Rafael Carmona-Paredes. Egresado de la Facultad de Ciencias (Física) de la UNAM en 1978. En 1983, obtuvo el grado de maestro en ingeniería (Control) y en 1992 el grado de doctor en ingeniería mecánica, ambos en la Facultad de Ingeniería de la UNAM. Trabaja en el Instituto de Ingeniería en proyectos sobre diseño y operación hidráulicos de conducciones de agua a presión, auspiciados por la Comisión Nacional del Agua. Es profesor en la Facultad de Ingeniería (licenciatura y posgrado). Ha dirigido 5 tesis de licenciatura y 2 de maestría. Desde 1987 es Investigador Nacional-SNI. En 1993, le fue otorgada la Distinción Universidad Nacional para Jóvenes Académicos y en 1995 el Premio de Investigación de la Academia Nacional de la Investigación Científica. 